\title{
Enhanced compositional sensitivity in atomic force microscopy by the excitation of the first two flexural modes
}

\author{
N. F. Martinez, S. Patil, J. R. Lozano, and R. Garcia ${ }^{a)}$ \\ Instituto de Microelectrónica de Madrid, CSIC, Isaac Newton 8, 28760 Tres Cantos, Madrid, Spain
}

(Received 27 June 2006; accepted 23 August 2006; published online 11 October 2006)

\begin{abstract}
The authors demonstrate that the compositional sensitivity of an atomic force microscope is enhanced by the simultaneous excitation of its first two flexural eigenmodes. The coupling of those modes by the nonlinear probe-surface interactions enables to map compositional changes in several conjugated molecular materials with a phase shift sensitivity that is about one order of magnitude higher than the one achieved in amplitude modulation atomic force microscopy. () 2006 American Institute of Physics. [DOI: 10.1063/1.2360894]
\end{abstract}

Simultaneous mapping of topography, chemical composition, and material properties of organic, biological, and heterogeneous surfaces is one of the goals of microscopic techniques. Amplitude modulation atomic force microscopy (AM-AFM) has provided very high resolution images of a large variety of materials and molecules such as nuclei acids, membrane proteins, polymer surfaces, silicon dots, or inorganic crystals. In AM-AFM a nanometer-size probe is excited at or near its free resonance frequency. The surface is imaged while the feedback electronics adjusts the tip-surface separation to keep a constant amplitude. ${ }^{1}$ The phase shift existing between the cantilever's excitation and its response has been applied to map compositional variations in heterogeneous surfaces. ${ }^{2-8}$ Phase contrast images are directly related to energy dissipation processes, consequently the achieved contrast increases with the amount of dissipated energy. ${ }^{9,10}$ This may involve the operation of the AM-AFM in the repulsive mode, i.e., in the presence of tip-sample mechanical contact. The resulting force applied on the sample could produce its irreversible deformation. In fact this has already been reported while imaging small biological molecules (antibodies). ${ }^{11}$

Rodriguez and García have recently proposed the simultaneous excitation of the first two flexural modes of the cantilever to increase the compositional sensitivity of an AFM while reducing the force exerted on the sample. ${ }^{12}$ The simulations showed that compositional contrast could be obtained in an attractive regime where the contrast was dominated by van der Waals forces in the $10-100 \mathrm{pN}$ range. Their approach goes beyond the standard point-mass models used to understand and explain AM-AFM. ${ }^{1,13,14}$ It implies the consideration of the AFM microcantilever as a continuous beam with many vibrational resonances. Related to the above approach is the use of higher harmonics of the fundamental resonance to extract information about material properties or to improve the instrument sensitivity. ${ }^{15-22}$ Stark et al. have demonstrated higher order harmonic imaging of heterogeneous samples. ${ }^{16}$ Sahin et al. have developed new rectangular cantilevers to exploit the sensitivity of higher harmonics to measure material properties. ${ }^{18}$ Balantekin and Atalar ${ }^{19}$ have imaged surfaces by using the third-harmonic amplitude, Crittenden et $a l^{20}$ have demonstrated that the higherharmonic resonances are sharper than the fundamental har-

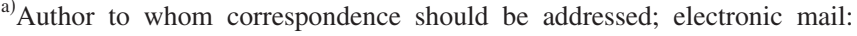
rgarcia@imm.cnm.csic.es monic, and Legleiler et al. have used higher harmonics to extract the time-resolved force interaction in fluids. ${ }^{21}$

In this letter we demonstrate that the sensitivity of an amplitude modulation AFM to map compositional changes in conjugated molecular materials can be enhanced by the simultaneous excitation of the first and second flexural modes. Under the same experimental conditions, the phase shift associated with the second mode is about one order of magnitude more sensitive to detect compositional variations than the phase shift of the first mode. Furthermore, the comparison between experiments and theory shows that the enhanced compositional sensitivity is achieved in a purely attractive and nondissipative regime.

The experiments are performed with a modified AM-AFM that enables the simultaneous excitation of the first and second resonances of the microcantilever beam (see Fig. 1). The rms amplitude of the first mode is fed back to the controller for topography imaging while the phase shifts of the first and second modes are recorded and plotted to detect compositional variations. Phase shift measurements are performed with doped $n^{+}$-type silicon cantilevers with a nominal radius of $10 \mathrm{~nm}$ (Nanosensors, Germany). The static force constant $k$, quality factors $\left(Q_{1}\right.$ and $\left.Q_{2}\right)$, and resonance frequencies $\left(f_{1}\right.$ and $\left.f_{2}\right)$ were, respectively, $2 \mathrm{~N} / \mathrm{m}, 173$ and $503 \mathrm{kHz}$, and 70.37 and $448 \mathrm{kHz}$ for imaging the tetrathiafulvalene derivatives, and $10 \mathrm{~N} / \mathrm{m}, 298$ and $752 \mathrm{kHz}$, and

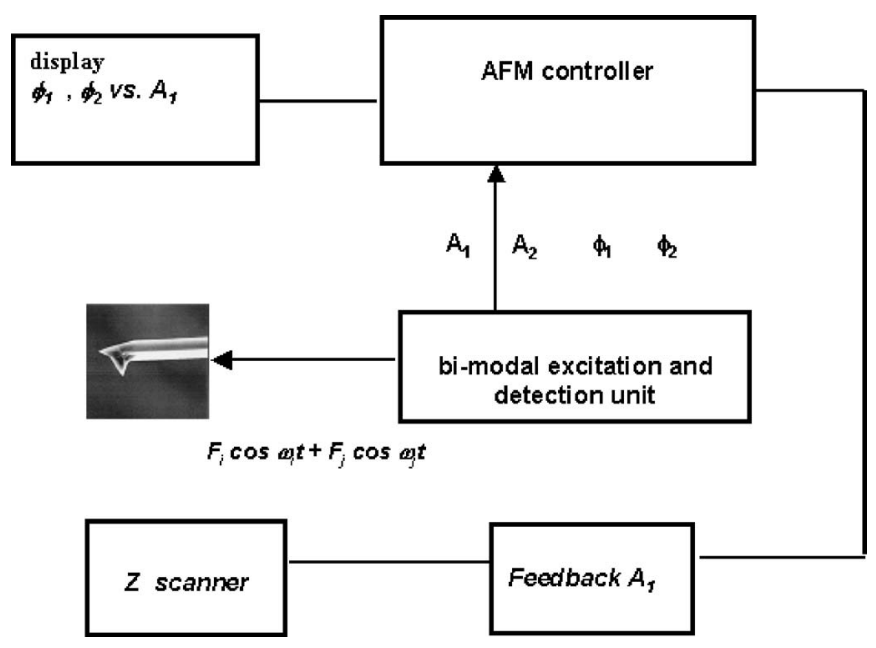

FIG. 1. Schematics of an amplitude modulation AFM operated under bimodal excitation. The cantilever is mechanically excited at its first two resonances. The photodiode signal (output) is processed to separate its components $A_{1}, A_{2}, \phi_{1}$, and $\phi_{2}$. 

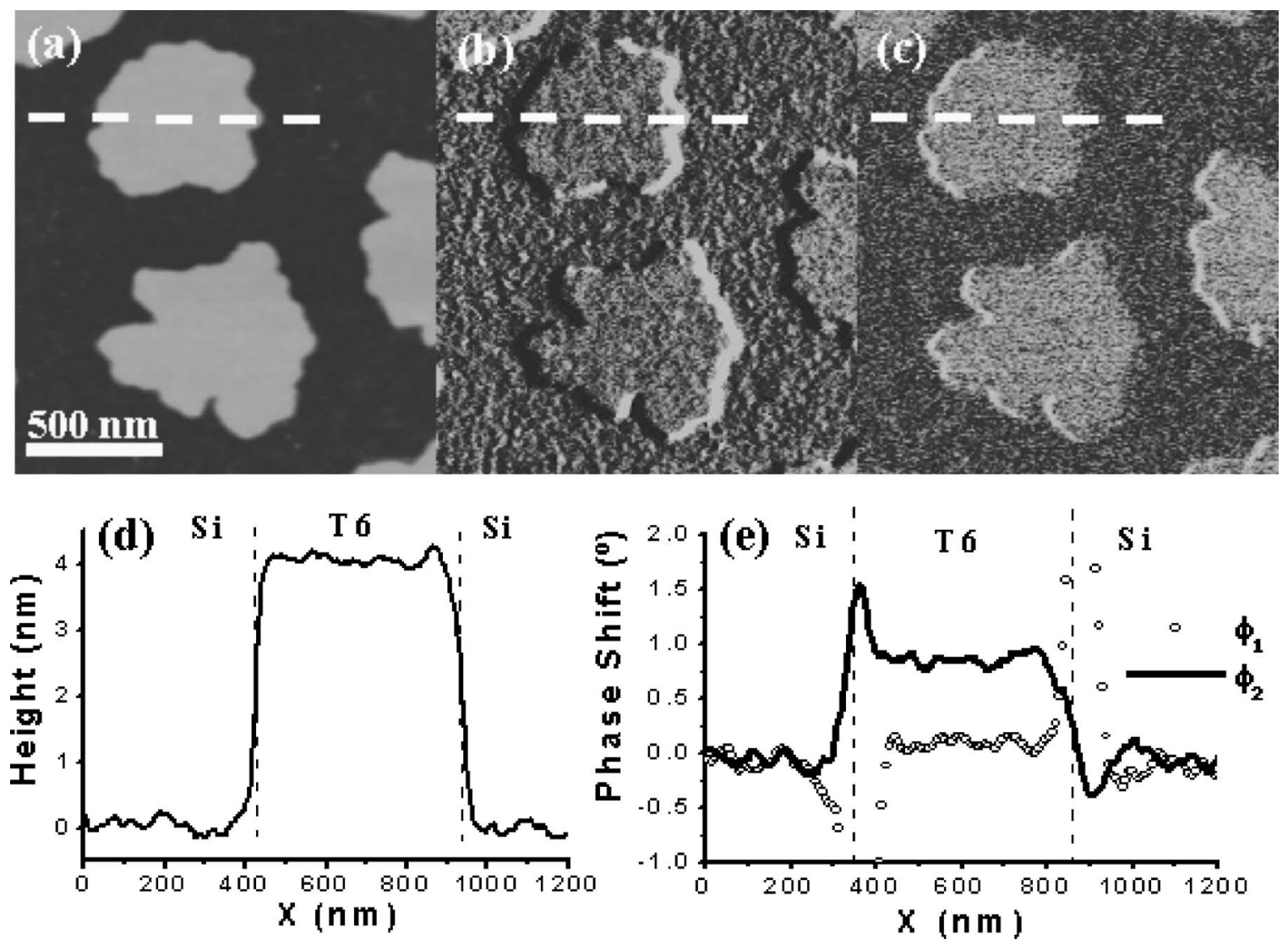

FIG. 2. Topography and phase images of several T6 islands on silicon: (a) topography, (b) first mode phase shift, and (c) second mode phase shift. (d) Height variation across the dashed lines shown in (a). (e) Phase shift variations across the dashed line shown in (b) and (c). The vertical discontinuous lines show the edges of the T6 island. The contrast observed between silicon and T6 with the phase shift of the first mode is barely above the noise level. T6 and silicon are clearly distinguished by plotting the phase shift of the second mode. $A_{1}=14 \mathrm{~nm}$ and $A_{2}=1.1 \mathrm{~nm} ; f_{1}=106.7 \mathrm{kHz}$ and $f_{2}=667.4 \mathrm{kHz} ; A_{\mathrm{sp}}=0.9 \mathrm{~A}$ rms.

\section{7 and $667.3 \mathrm{kHz}$ for imaging sexithienyl molecules.}

The semiconductor samples were $p$-type $\operatorname{Si}(100)$ with a resistivity of $0.1-1.4 \Omega \mathrm{cm}$. The silicon surface is chemically treated to be covered by a very thin silicon dioxide film $(\sim 0.6 \mathrm{~nm})$. A solution of tetrathiafulvalene (TTF) derivatives of $10^{-4} \mathrm{M}$ in acetonitrile was deposited by drop casting on silicon surfaces. ${ }^{23}$ Sexithienyl (T6) molecules were deposited onto silicon substrates by sublimation in ultrahigh vacuum of the polycrystalline material from a Knudsen cell in an organic molecular beam deposition apparatus. In order to obtain the formation of two-dimensional ordered T6 layers, high substrate temperature during deposition and low deposition rate and film thickness were chosen on the basis of the T6 film "kinetic" phase diagram. ${ }^{24}$

The simulations were performed by modeling the threedimensional microcantilever as a rectangular beam and applying the Euler-Bernoulli equation as it is described in Ref. 25.

Figure 1 shows schematics of the experimental setup to perform the simultaneous excitation and the phase shift representation of the first two normal modes. The key element is the simultaneous excitation of the microcantilever to its first and second resonances by a mechanical force described by

$$
F_{\text {exc }}(t)=F_{1} \cos \omega_{1} t+F_{2} \cos \omega_{2} t,
$$

where $\omega_{1}$ and $\omega_{2}$ are the angular frequencies of the first and second flexural modes of the free cantilever.

The output signal of the first mode is used to image the topography of the sample while the phase shifts of both first Downloaded 22 Oct 2009 to 161.111.235.169. Redistribution subject and second modes are used to map changes in the composition of the atoms or molecules under the tip.

Figure 2 shows the topography and phase shift images of several T6 monolayers deposited on a silicon surface. Topography and phase images are taken simultaneously. The size and shape of the T6 islands on silicon make them ideal samples to test the compositional sensitivity of an AFM. Figures 2(b) and 2(c) show the phase images of the T6 islands on silicon by recording, respectively, the phase shift variation of the first and second modes. The cross section along the dashed line in Fig. 2(b) shows a $\Delta \phi_{1}=0.1^{\circ}$ contrast between the silicon and T6 [Fig. 2(e)], i.e., barely above the noise level $\left(\sim 0.05^{\circ}\right)$. On the other hand, the phase shift difference obtained in the second mode is $\Delta \phi_{2}=0.9^{\circ}$ [Fig. 2(e)]. The comparison between $\phi_{1}$ and $\phi_{2}$ cross sections shows that the material contrast is enhanced by a factor of 10 by plotting the second mode phase shift. The peaks that appear in the phase shift cross sections are topographic artifacts due to the finite time response of the feedback electronics. We remark that the first and second mode phase images have been obtained under exactly the same conditions and forces.

The general character of the method is demonstrated by imaging an organic compound with different chemical composition and shape. Figure 3(a) shows the topography of a cluster of conjugated molecules (TTF derivative) deposited on a silicon surface. The first mode phase image (not shown) and cross section [Fig. 3(b)] do not show any kind of contrast other than the topography artifacts due to the feedback electronics finite time response. On the other hand, the secto AlP license or copyright; see http://apl.aip.org/apl/copyright.jsp 

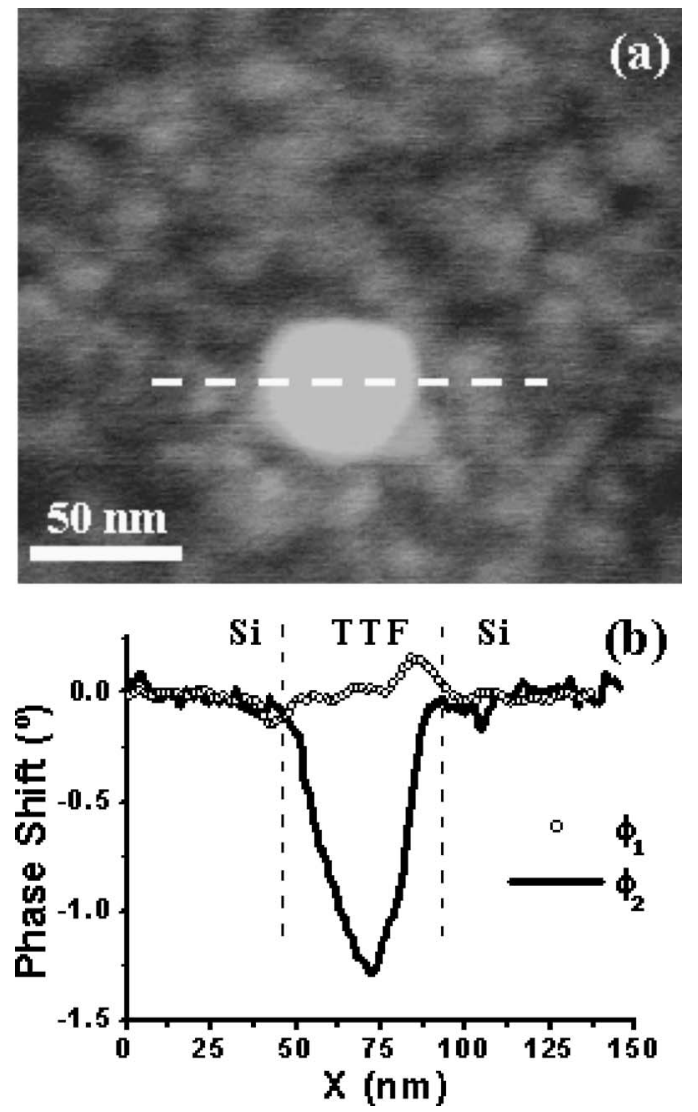

FIG. 3. (a) AM-AFM topography of a cluster of conjugated molecules (TTF). (b) First and second mode phase shifts across the dashed line shown in (a). $A_{1}=23 \mathrm{~nm}$ and $A_{2}=0.5 \mathrm{~nm} ; f_{1}=70.35 \mathrm{kHz}$ and $f_{2}=448.19 \mathrm{kHz} ; A_{\mathrm{sp}}$ $=0.9 A_{\text {rms }}$.

ond mode phase shift cross section shows a noticeable $\Delta \phi_{2}$ $=1.2^{\circ}$ change between the cluster of molecules and the silicon surface [Fig. 3(b)].

The comparison between theory and experiments for T6 monolayers is shown in Table I for a long-range tip-surface interaction described by the following equation:

$$
F_{\mathrm{vdW}}=-\frac{H R}{6 d^{2}}
$$

where $H, R$, and $d$ are the Hamaker constant, tip's radius, and tip-surface instantaneous distance. We have taken Hamaker constants for the silicon dioxide-air-silicon dioxide and T6air-silicon dioxide interfaces as $H=6.7 \times 10^{-20}$ and 4.5 $\times 10^{-20} \mathrm{~J}$, respectively $(R=10 \mathrm{~nm})$. Those values have been derived by following Ref. 26. The model predicts the observed experimental trend although it overestimates the enhancement of the phase shift of both modes. This could be attributed to several factors such as the strength of the internal damping in the experimental cantilever, which is currently unknown. The internal damping could decrease the coupling between the modes and consequently the enhance-

TABLE I. Theory and experiment comparison for first and second mode phase shift cross sections obtained on T6 monolayers deposited on silicon.

\begin{tabular}{lcc}
\hline \hline & $\begin{array}{c}\Delta \phi_{1}(\mathrm{deg}) \\
\phi_{1}(\mathrm{~T} 6)-\phi_{1}(\mathrm{Si})\end{array}$ & $\begin{array}{c}\Delta \phi_{2}(\mathrm{deg}) \\
\phi_{2}(\mathrm{~T} 6)-\phi_{2}(\mathrm{Si})\end{array}$ \\
\hline Experiments & 0.1 & 0.9 \\
Simulations & 0.22 & 3.87 \\
\hline
\end{tabular}

ment of the phase shift signal of the second mode. The differences existing in the geometry between the simulated and the experimental cantilever could also contribute to the observed numerical discrepancies. The experimental cantilever has a trapezoidal section while the simulated beam is rectangular. The coupling between modes also gives rise to a small phase shift in the first mode. We remark that this happens in the absence of any dissipative process at the tip-sample interface.

In short we have demonstrated the compatibility of topographic and compositional mapping of an AFM under the excitation of its first two flexural modes. We have demonstrated that the phase shift associated with the second mode is one order of magnitude more sensitive to detect compositional variations in conjugated molecular materials than the one of the first mode. These results confirm previous simulations that predicted a higher sensitivity in atomic force microscopy by the simultaneous excitation of the first two flexural modes. The present results together with the theoretical simulations pave the way to develop a spatially resolved force spectroscopy with atomic or nanometer resolution for operation in liquids or air.

The authors thank Fabio Biscarini, Neil Oxtoby, and Concepció Rovira for providing the conjugated molecules. This work was financially supported by the European Commission (FORCETOOL, NMP4-CT-2004-013684).

${ }^{1}$ A. S. Paulo and R. Garcia, Phys. Rev. B 66, 041406 (2002).

${ }^{2}$ R. Garcia, J. Tamayo, and A. San Paulo, Surf. Interface Anal. 27, 312 (1999).

${ }^{3}$ G. Reiter, G. Castelein, J. U. Sommer, A. Röttele, and T. Thurn-Albrecht, Phys. Rev. Lett. 87, 226101 (2001).

${ }^{4}$ M. Stark, C. Möller, D. J. Muller, and R. Guckenberger, Biophys. J. 80, 3009 (2001).

${ }^{5}$ M. S. Marcus, R. W. Carpick, D. Sasaki, and M. A. Eriksson, Phys. Rev. Lett. 88, 226103 (2002)

${ }^{6}$ W. Wu, K. Matyjaszewski, and T. Kowalewski, Langmuir 21, 1143 (2005).

${ }^{7}$ N. Rehse, S. Marr, S. Scherdel, and R. Magerle, Adv. Mater. (Weinheim, Ger.) 17, 2203 (2005).

${ }^{8}$ Z. Suo, X. Yang, R. Avci, L. Kellerman, D. W. Pascual, M. Fries, and A. Steele, Biophys. J. (2006).

${ }^{9}$ P. D. Ashby and C. M. Lieber, J. Am. Chem. Soc. 127, 6814 (2005).

${ }^{10}$ N. F. Martinez and R. Garcia, Nanotechnology 17, S167 (2006).

${ }^{11}$ A. San Paulo and R. García, Biophys. J. 78, 1599 (2000).

${ }^{12}$ T. R. Rodriguez and R. García, Appl. Phys. Lett. 84, 449 (2004).

${ }^{13}$ P. Martin, S. Marsaudon, J. P. Aimé, and B. Bennetau, Nanotechnology 16, 901 (2005).

${ }^{14}$ S. I. Lee, S. W. Howell, A. Raman, and R. Reifenberger, Phys. Rev. B 66, 115409 (2002).

${ }^{15}$ R. W. Stark and W. Heckl, Rev. Sci. Instrum. 74, 5111 (2003).

${ }^{16}$ M. Stark, R. W. Stark, W. Heckl, and R. Guckenberger, Appl. Phys. Lett. 77, 3293 (2000).

${ }^{17}$ O. Sahin, G. Yaralioglu, R. Grow, S. F. Zappe, A. Atalar, C. Quate, and O. Solgaard, Sens. Actuators, A 114, 183 (2004).

${ }^{18}$ O. Sahin, C. F. Quate, O. Sogaard, and A. Atalar, Phys. Rev. B 69, 165416 (2004).

${ }^{19}$ M. Balantekin and A. Atalar, Appl. Phys. Lett. 87, 243513 (2005).

${ }^{20}$ S. Crittenden, A. Raman, and R. Reifenberger, Phys. Rev. B 72, 235422 (2005).

${ }^{21}$ J. Legleiter, M. Park, B. Cusik, and T. Kowalewski, Proc. Natl. Acad. Sci. U.S.A. 103, 4813 (2006).

${ }^{22} \mathrm{R}$. Proksch (private communication).

${ }^{23}$ M. Mas-Torrent and C. Rovira, J. Mater. Chem. 16, 433 (2006).

${ }^{24}$ M. Muccini, M. Murgia, F. Biscarini, and C. Taliani, Adv. Mater. (Weinheim, Ger.) 13, 355 (2001).

${ }^{25}$ T. R. Rodriguez and R. Garcia, Appl. Phys. Lett. 80, 1646 (2002).

${ }^{26}$ J. Israelachvili, Intermolecular and Surface Forces (Academic, London, 1992). 